(2) Open Access Full Text Article

REVIEW

\title{
Impact of Access Cavity Design on Fracture Resistance of Endodontically Treated Molars: A Systematic Review
}

This article was published in the following Dove Press journal: Clinical, Cosmetic and Investigational Dentistry

\author{
Musab Saeed' \\ Mona Al-Obadi $\mathbb{D D}^{2}$ \\ Asma Salim² \\ Alaa Y Alsawaf $^{2}$ \\ Karrar Hadi (iD ${ }^{3}$ \\ 'Department of Clinical Sciences, Ajman \\ University, Ajman, United Arab Emirates; \\ ${ }^{2}$ Ajman University, Ajman, United Arab \\ Emirates; ${ }^{3}$ College of Dentistry, Ajman \\ University, Ajman, United Arab Emirates
}

Correspondence: Musab Saeed Department of Clinical Sciences, Ajman University, Ajman, United Arab Emirates Tel +971670562I I

Email m.saeed@ajman.ac.ae
Objective: The main aim of this present systematic review is to ascertain whether a conservative endodontic cavity (ConsAC) has a better fracture resistance than a traditional access cavity (TradAC) in endodontically treated molars.

Materials and Methods: Three independent reviewers researched without limits in multiple engines: PubMed, ScienceDirect, Scopus, ResearchGate, EBSCOhost, Wiley Online Library, and Google Scholar, in addition to reference and hand search. Articles were chosen according to a certain inclusion and exclusion criteria, which, in brief, are in vitro studies assessing the fracture resistance difference between ConsAC and TradAC performed on extracted sound human molar teeth. A quality assessment criteria was produced to evaluate the chosen articles and categorized them according to their risk of bias into low, moderate and high.

Results: Out of a total of 105 studies, which were obtained from seven different search engines, only eight in vitro studies were included after eliminating the duplicates followed by the application of the eligibility criteria. Five of the articles showed low risk of bias while the others revealed a moderate risk of bias.

Conclusion: To sum up, there is insufficient evidence to give a final decision whether ConsAC is more advantageous than TradAC in terms of fracture resistance and further studies regarding this topic are needed.

Keywords: traditional access cavity, conservative access cavity, endodontically treated teeth, molars, fracture resistance

\section{Introduction}

Endodontically treated teeth were found to have the worse long-term survival rate that results in reduced fracture resistance when compared to non-endodontically treated teeth. ${ }^{1-5}$ Thus, many clinical studies were drawn to the fact that Root Canal Treatment (RCT) is a causative factor for tooth fracture, produced by loss or reduction in tooth structure due to caries removal and preparation of the access cavity, and/or extreme removal of root dentine during the shaping and instrumentation process. ${ }^{4-8}$

In addition to that, it has been proposed that the most critical factor regarding fracture resistance and survival of endodontically treated teeth is the amount of remaining dentine. Loss of tooth structure during preparation includes anatomic structures such as ridges, cusps, and the pulp chamber roof., 5,10

Traditional endodontic access cavity (TEC) involves straight-line pathways into the canals by preparing through caries and existing restorations, reaching to the 
apical constriction by extending the orifices of the root canal through the removal of cervical dentin projections, while only maintaining the remaining sound tooth structures. ${ }^{2,11,12}$

The peri-cervical dentin, an area roughly $4 \mathrm{~mm}$ above the alveolar bone crest and $6 \mathrm{~mm}$ below it, was found to be a critical factor in regards to the long-term survivability and optimum function, ${ }^{13}$ acting as medium to aid in the transmission of forces between the occlusal aspect and the $\operatorname{root}(\mathrm{s}){ }^{11}$

Increasing cuspal flexure during TEC by peri-cervical dentin removal can negatively affect the mechanical and biological responses of teeth resulting in increased stress at the crown portion and the surfaces of roots, which in sequence may increase the liability of endodontic treated teeth to fracture when exposed to functional loads. ${ }^{14-18}$

According to Clark et al, maintaining 0.5 to $3 \mathrm{~mm}$ of the roof of the pulp chamber is the safest approach to prevent inflicting damage to this dentin, which would reduce the cusp flection and, therefore, the index of fracture of the tooth. ${ }^{19}$

Recently, the minimally invasive endodontics concept is obtaining acceptance gradually within clinical dentistry, although there is only limited scientific evidence that is in favor of this concept. ${ }^{13}$ In minimally invasive endodontics, which is also termed as conservative or contracted endodontic access cavity (CEC) design is mostly focused on preserving as much as possible of the coronal tooth structure by reducing the dimensions of access cavities and ignoring the traditional needs of a straightline access. It, CEC, is considered as a substitute to TEC, as it prefers the removal of restorative materials instead of enamel or dentin and of removal of occlusal structures instead of cervical dentin. It maintains parts of the pulp chamber's roof while emphasizing the importance of protecting the peri-cervical dentin, which results in conserving the mechanical stability, and subsequently, prolong the life and improve function of the treated teeth. $^{11-13}$

Nowadays, the traditional requisites of the endodontic access cavity preparation is decreasing mainly due to improvements in the various fields that help clinicians to shape the access cavity to be as conservative as possible while maintaining teeth structures as much as possible. They include improvements in endodontic instruments, imaging, visual enhancers (optical magnification), and clinical microscopes. $^{20,21}$
On the other hand, this relatively modern cavity design may restrict the irrigation, instrumentation, and obturation of root canals. An insufficiently sized access cavity also increases the incidence of procedural errors during endodontic treatment. ${ }^{21}$

In the latest study regarding this concept which was done by Silva et al 2020, they proposed a universal nomenclature for the current status on minimal access cavity preparations. They divided them into six groups: traditional access cavity (TradAC), conservative access cavity (ConsAC), ultra-conservative access cavity (UltraAC), truss access cavity (TrussAC), caries-driven access cavity (CariesAC), and restorative-driven access cavity (RestoAC). ${ }^{22}$

In earlier laboratory studies, many researchers that compared ConsACs with TradACs, have found ConsACs enhanced the fracture resistance under a constant load. However, other studies do not show a significant difference between ConsACs and TradACs in preserving fracture strength. ${ }^{2}$ Therefore, the findings pertaining to the effect of ConsACs in relation to fracture resistance remains controversial. Thus, this systematic review is prepared to be able to retort whether conservative endodontic "access" cavities (ConsACs) enhance the fracture resistance of molars when compared to the traditional method.

\section{Materials and Methods \\ Study Design}

All studies from 2010 onwards that measured the impact of access cavity with outlines being fracture resistance in extracted humanoid molars were systematically reviewed. A controlled group of TradACs was used for comparison. The current systematic review was conducted during January- May 2020 in accordance with the Preferred Reporting Items for Systematic Reviews (PRISMA) guidelines.

\section{Literature Search Strategy}

Three independent reviewers performed a systematic search to find all the published studies in the most popular electronic databases: Scopus, PubMed, ResearchGate, EBSCOhost, ScienceDirect, Wiley Online Library, and Google Scholar having set the publication date range from January 2010 to January 2020. The keywords were selected using terms of Medical Subject Heading (MeSH) and text word (tw) terms or a mixture of both: "endodontic access design" (MeSH), "endodontically treated tooth" 
(MeSH), "molars" (tw), "conservative access cavity" (MeSH), "traditional access cavity" (MeSH), "fracture resistance" (tw), "fracture strength" (tw), and "minimally invasive endodontics" (MeSH). The "OR" and "AND" Boolean operators were used to combine keywords. Additional search was done by using the "related citations" feature in ResearchGate.

\section{Eligibility Criteria}

To be eligible for inclusion, the studies should have a main aim of evaluating the effect of endodontic access cavity designs on fracture resistance by comparing conservative endodontic cavities (ConsACs) with traditional endodontic cavities (TradACs) having a sample size between 30-60 molar teeth to make inferences about a population from a sample. The eligibility criteria follows the PICO scheme: The human molar teeth included in the studies are to be extracted with fully formed (mature) apex (P-participants), conservative endodontic cavities (ConsACs) method (Iintervention), comparison groups of traditional endodontic cavities (TradACs) method (C-comparison), and the data of the resistance to fracture as an outcome (O-outcome). Though the PICO scheme is commonly used for clinical trials, an intervention was presented in $100 \%$ of the in vitro studies included in this work. Moreover, all the included studies should be published after January 2010 and written in English. Excluded studies comprised all literature reviews, opinion articles, letters, serial case, case reports, conference abstracts, and those who involved immature or artificial teeth in their studies.

\section{Study Selection}

There are three different reviewers. In the first stage, all reviewers start by evaluating the headings and abstracts of selected articles. Next, they applied the eligibility criteria, in which the title of articles that did not contain adequate information for a definitive decision was retrieved and examined entirely. In the second stage, the selected papers were separately revised and screened by the same reviewers. Disagreements between the reviewers on whether to accept or reject a study were discussed thoughtfully until a consensus was achieved. A fourth reviewer resolved any discrepancies in cases where no consensus was obtained. Later, full-text evaluation of the potentially relevant studies to be included in this systematic review. Duplication of an article was eliminated.

\section{Data Collection}

Three reviewers performed data extraction in all the included studies independently. Any potential conflict was resolved by discussion with a fourth reviewer. The following data was pulled out from all studies and noted: study characteristics (name of the authors, year of publication, and country where the study took place), sample characteristics (type of the tooth chosen, sample size and sample per group), endodontic procedures (access cavity design, technique, obturation and restoration), and fracture resistance (mean load at failure and the analysis similar to the article).

\section{Quality Assessment}

Assessment of the chosen studies was done in accordance with the methods used in previous systematic reviews concerning in vitro studies. ${ }^{11,23,24}$ The methodological quality of the included studies was assessed independently by the same three reviewers using the following parameters: (1) sample size calculation, (2) samples with similar dimensions, (3) presence of a control group (TradAC teeth), (4) execution of filling procedures, (5) presence of coronal restoration, and (6) correct statistical analysis carried out. Since shapes of the access cavity outlines vary and it is easier for the operator to identify the performed treatment, no blinding of the operator was taken into consideration. "Yes" and "No" were assigned for the parameters reported or missing in original studies respectively. Articles to be classified as a low risk of bias if they report five or six items, a moderate risk of bias in case of three to four items reported, and a high risk of bias is deemed when only one or two items reported. A fourth reviewer resolved any disagreement between the reviewers.

\section{Results \\ Study Selection}

The detailed process of the literature search and article screening is defined in Figure 1. Initial screening of electronic databases yielded a total of 108 publications. The engines used as sources for publications were PubMed (n =27), ScienceDirect $(n=17)$, Researchgate $(n=35)$, Scopus $(\mathrm{n}=8)$, EBSCO $(\mathrm{n}=15)$, Wiley $(\mathrm{n}=5)$, Google scholar $(n=1)$, and an addition of three articles identified from the references. We ended up with fifty-nine articles after removing the duplicates. Thirty-three articles were read in full text for eligibility after the exclusion of twenty-six articles by title and abstract ineligibility. Out 


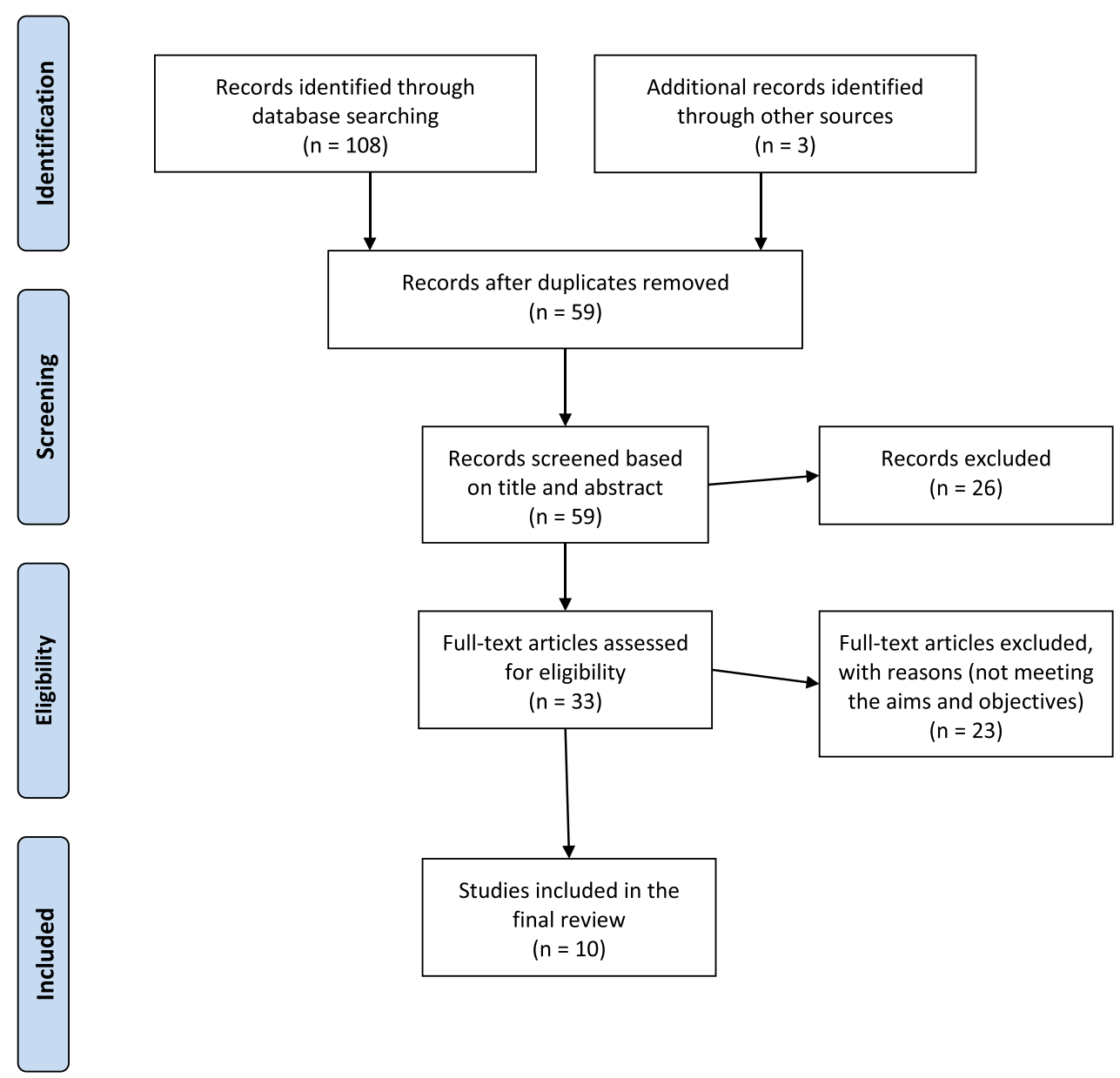

Figure I Prisma 2009 flowchart diagram of literature search outlining the study identification and screening process. Adapted from Moher D, Liberati A, Tetzlaff J, Altman DG, The PRISMA Group (2009). Preferred Reporting Items for Systematic Reviews and Meta-Analyses: The PRISMA Statement. PLoS Med 6(7): el000097. doi:I0.137I/ journal.pmed $000097 .^{25}$ Creative Commons.

of thirty-three articles left, twenty-three articles were excluded due to the fact that the case study design was not cross sectional, not including a TradAC for comparison, studies not involving molar teeth, studies using finite element analysis, not fulfilling the target of our study such as access cavity and fracture resistance, and/or sample size beyond the selected range. Finally, ten articles were included in this systematic review, having fulfilled the inclusion criteria.

\section{Study Characteristics}

All accepted articles studied the impact of conservative access cavities on fracture resistance in extracted molars and compared it to traditional access cavities. Studies used different sample sizes ranging from 30 to 60 . Some studies did not perform sample size calculation. ${ }^{21,26-28}$ Earlier to the loading test, few studies did not perform a filling of the canals, or restoration procedures, but they did that for all the groups to ensure standardizations. ${ }^{5,21,29}$ Statistical analyses were carried out by all the included studies, some showed statistically significant results when comparing ConsAC to TradAC, and others found no statistical differences between them.

\section{Fracture Resistance Results of Individual Studies}

A Summary of descriptive characteristics of included studies is shown in Table 1. Corsentino et al in 2018 observed no significant differences between TradAC, ConsAC and control groups. I.A. Osman and H.A Ahmed in 2018 finalized that the fracture resistance of ConsAC was statistically significantly higher in mandibular molars compared to TradAC groups. While there was no significant difference from the control group (sound molars). On the other hand, maxillary molars show no statistically significant differences in fracture strength 







\begin{tabular}{|c|c|c|c|}
\hline 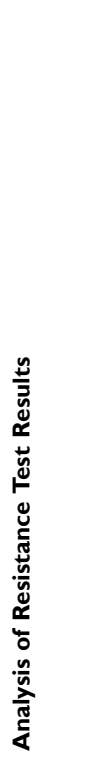 & 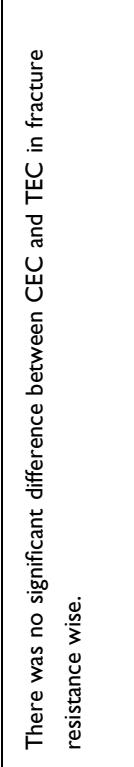 & 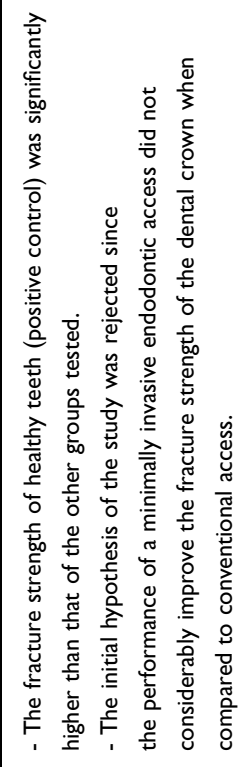 & 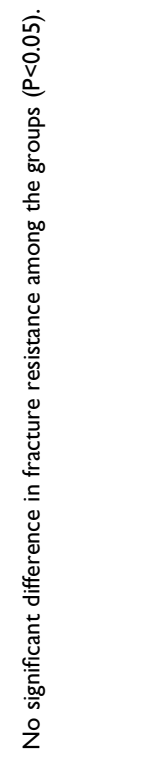 \\
\hline 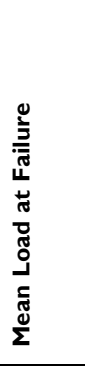 & 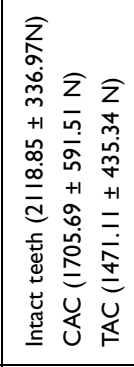 & 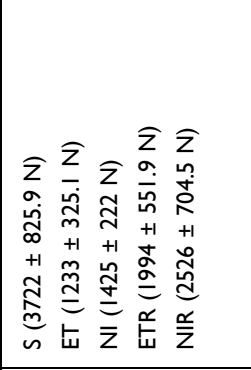 & 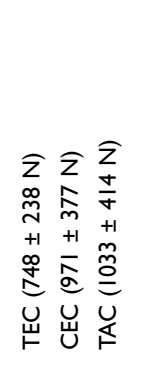 \\
\hline 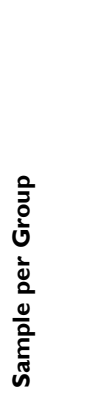 & 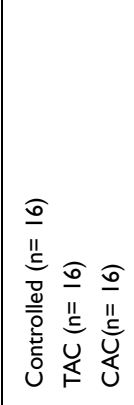 & 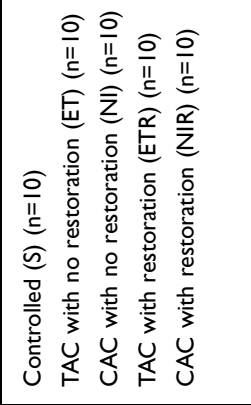 & 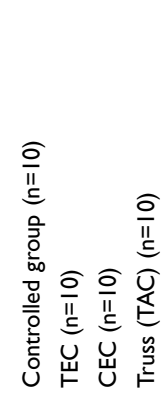 \\
\hline 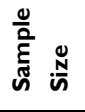 & \begin{tabular}{|l} 
\\
ơ \\
II \\
\end{tabular} & $\begin{array}{l}0 \\
\text { on } \\
11 \\
=\end{array}$ & $\begin{array}{l}q \\
11 \\
c\end{array}$ \\
\hline & 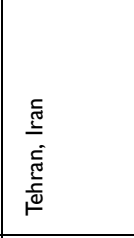 & 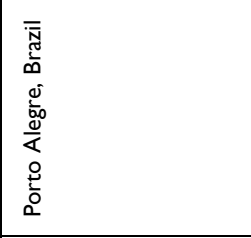 & 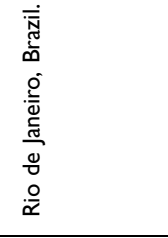 \\
\hline 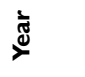 & $\frac{\infty}{2}$ & ๙ิ & ๙ิ \\
\hline $\begin{array}{l}\frac{n}{2} \\
\frac{\dot{t}}{2} \\
\frac{1}{4}\end{array}$ & 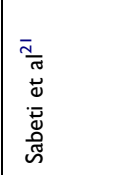 & 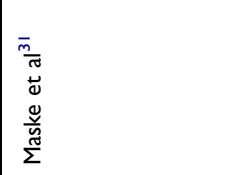 & 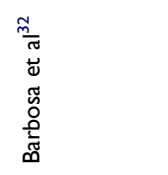 \\
\hline
\end{tabular}

between ConsAC group and TradAC group, nor between ConsAC and sound control group. However, fracture strength of TradAC was significantly lower than that of the control group. Krishan et al in 2014 found that the ConsAC in mandibular molars has higher resistance to fracture. Makati et al in 2018 found a significant difference between TradAC and ConsAC. Moore et al in 2016 found that the fracture resistance did not differ significantly between the ConsAC and TradAC group but when compared with the intact teeth group both showed a significantly lower fracture resistance. Plotino et al in 2017 found that the mean load at fracture in the TradAC group was significantly lower than the intact and ConsAC groups, whereas observed no difference among the control and ConsAC groups. Rover et al in 2017 showed that there is no statistical difference between TradAC and ConsAC groups in the mean load at failure. ${ }^{30}$ Sabeti et al in 2018 observed that ConsAC group was not much better than the TradAC group in fracture resistance. Maske et al in 2020 found that there was a significant difference between healthy intact teeth compared with the other groups, but no difference between TradAC and ConsAC groups in fifty lower third molars. ${ }^{31}$ Lastly, Barbosa et al in 2020 got similar results to Maske et al when he compared TradAC to ConsAC on forty mandibular molars. ${ }^{32}$

\section{Study Quality Assessment}

According to the considered parameters that have been used for the quality assessment and risk of bias, out of the ten studies included, three were of moderate risk of bias (I.A. Osman, H.A Ahmed 2018, Krishan et al 2014, Sabeti et al 2018) and the rest were deemed as low risk of bias as shown in Table 2.

\section{Discussion}

Many studies have found that the main causative factor making the tooth brittle and more susceptible to fracture is the loss of its sound structure during endodontic access preparation. In the traditional endodontic access cavity, the concept of "extension for prevention" in endodontic access is followed to facilitate the procedure with no dentin interfering to obtain a straight-line access. ${ }^{12}$ However; it removes a significant amount of tooth structure. According to this, the conservative endodontic access cavity concept is targeting the preservation of occlusal tooth structure by reducing the access cavity. ${ }^{9,13}$ Since then, concern for this cavity design has increased in this speciality, this 
Table 2 Quality Assessment and Risk of Bias

\begin{tabular}{|l|l|l|l|l|l|l|l|}
\hline Study & $\begin{array}{l}\text { Sample } \\
\text { Size } \\
\text { Calculation }\end{array}$ & $\begin{array}{l}\text { Samples } \\
\text { with } \\
\text { Similar } \\
\text { Dimensions }\end{array}$ & $\begin{array}{l}\text { Control Group } \\
\text { (Intact Teeth, } \\
\text { or } \\
\text { Conventional } \\
\text { Access })\end{array}$ & $\begin{array}{l}\text { Performance } \\
\text { of Filling } \\
\text { Procedures }\end{array}$ & $\begin{array}{l}\text { Performance } \\
\text { of } \\
\text { Restoration } \\
\text { Procedures }\end{array}$ & $\begin{array}{l}\text { Statistical } \\
\text { Analysis } \\
\text { Carried } \\
\text { Out }\end{array}$ & $\begin{array}{l}\text { Risk of } \\
\text { Bias }\end{array}$ \\
\hline Corsentino et al & No & Yes & Yes & Yes & Yes & Yes \\
\hline I.A. Osman, H.A Ahmed & Yes & Yes & Yes & No & No & Yes & Moderate \\
\hline Krishan et al ${ }^{29}$ & Yes & Yes & Yes & No & No & Yes \\
\hline Makati et al ${ }^{27}$ & No & Yes & Yes & Yes & Yes & Yes \\
\hline Moore et al ${ }^{14}$ & Yes & Yes & Yes & Yes & Yes \\
\hline Plotino et al ${ }^{28}$ & No & Yes & Yes & Yes & Low \\
\hline Rover et al ${ }^{30}$ & Yes & Yes & Yes & Yes & Yes & Yes \\
\hline Sabeti et al ${ }^{21}$ & No & Yes & Yes & Yes & Yes & No & Yes \\
\hline Maske et al ${ }^{31}$ & Yes & Yes & Yes & Yes & Yes & Yes & Low \\
\hline Barbosa et al & Yes & & Yes & Low \\
\hline
\end{tabular}

systematic review of the in vitro studies is to assess the influence of the ConsAC on the human molars' resistance to fracture.

From all studies $(n=105)$ which were obtained via electronic search from seven different search engines, we found that only ten of them were included after elimination of duplication and application of the eligibility criteria. The ten included studies were classified as low/ moderate risk of bias. In this systematic review, Only 4 of the included studies showed an enhanced fracture resistance of ConsACs compared to TradACs. ${ }^{5,27-29}$

Makati et al in 2018 showed that molars with ConsAC group had statistically higher fracture resistance with a mean difference of 1662.07 N. Plotino et al in 2017 revealed that the mean load at fracture for teeth in the ConsAC group was considerably higher than the TradAC and concluded that ConsAC had comparatively higher fracture resistance. I.A. Osman et al in 2018 found that the fracture resistance was significantly higher in mandibular molars in ConsAC compared to traditional access cavity (TAC) groups, while no considerable difference in fracture resistance in maxillary molars was found. Krishan et al in 2014 showed that the average load at fracture for molars with ConsAC was significantly higher than the TradAC. The authors in the last two studies ${ }^{5,29}$ performed the fracture loading test without obturating and restoring the treated teeth, which rendered them a moderate risk of bias. Additionally, it is recognized that performing a final filling of endodontically treated teeth reinstates the fracture resistance up to $72 \%$ of that of intact teeth. ${ }^{14,33}$ However, we accepted these two articles because they have tested all the groups without obturating the canals or applying a restorative material to seal the access cavity.

On the other hand, the remaining six studies (Corsentino G. et al 2018, Sabeti M. et al 2018, Moore B. et al 2016, Rover G. et al 2017, Maske et al 2020 and Barbosa et al 2020) ascertained a similar conclusion and results as Ozyurek et al in 2018 who found that there is no major change in fracture resistance when performing TradAC or ConsAC. ${ }^{34}$

In a systematic review done by Silva et al in 2017, they evaluated fracture resistance of ConsAC with TradAC for any group of teeth (incisors, premolars, and molars). Six articles met their inclusion criteria out of 180 articles. Three of which had low risk of bias while the other three had a moderate risk of bias. Silva et al found that half of the included articles showed no significant difference between the two groups while the other half showed an enhancement in fracture resistance when performing ConsAC when compared to performing TradAC. ${ }^{11}$

Minimally invasive dentistry interventions highlighted the preservation of tooth structure by avoiding un-roofing of the pulp chamber and avoiding over-flaring of canal 
orifices as well as avoiding aggressive dentine removal for shaping. ${ }^{35-37}$ However, ConsAC can primarily be accomplished on intact teeth, which does not indicate the clinical daily routine since most available scenarios are treating carious or defective teeth. Authors reported only $8 \%$ of intact teeth treated cases in the previous five years. ${ }^{28}$

Moreover, ConsAC's risks outweigh its benefits, through raising the likelihood of missed canals. In addition, since the main drive of endodontic treatment is to eradicate and destroy bacteria present in a diseased pulp and having studies revealing that bacteria can infiltrate into and colonize almost half the length of dentinal tubules, ConsAC will be negatively impacting the instrumentation efficacy, which is a great concern. ${ }^{11,14,21}$

A recent study by Barbosa et al in 2020 evaluated the quality of root canal disinfection following the ConsAC procedure and obtained larger percentages of unprepared canal surface area and a larger volume of remaining root filling material within the pulp chamber. ${ }^{32}$

Clinically, the ConsAC procedure faces clinicians with many challenges. The main challenge in molar teeth is the accessibility to the offending tooth. ConsAC could be difficult, if not impossible in a case of limited accessibility. ${ }^{5}$ Other challenges which may face the clinicians in the term of difficulty are detecting all canals, and removing pulp tissues from pulp horns, removing debris, and necrotic material, and at the same time any procedural complication(s) that may arise due to limited access cavity. ${ }^{29}$ All the risks and difficulties surrounding Cons AC obligate clinicians to obtain certain skills for the standard use of this technique and focus on claiming the benefits like increasing the fracture resistance while dismissing the risks of comprising the prognosis. ${ }^{14}$

For a long time, it has been known that not all research designs are comparable in the risk of error and bias in their results. For instance, a randomized controlled trial is believed to provide the most reliable evidence when assessing the effectiveness of an intervention because the processes used tends to minimize the risk of confounding influences affecting the results. ${ }^{36}$ Since clinical trials accomplish evidence with higher strength, it can directly influence institutional policies. Subsequently, it provides new education models amongst academic organizations in order to develop the most proficient procedure for treating the patients. However, clinical research needs high workforce and costs. Thus, it is important to assess the cost-benefit before starting the project. ${ }^{38}$ Unfortunately, not a single randomized clinical trial found in the electronic search covered this topic.
On the other side, those in vitro studies do not mimic the clinical oral condition present in real life. For example, the used loading to fracture methodology in vitro analyses does not exactly imitate intraoral conditions. ${ }^{11,28}$ Intraorally, conditions like irreproducible oral hygiene status, cariogenic and erosive challenges, masticatory forces, and other variables are difficult to be mimicked in laboratory studies. ${ }^{11,39}$

However, in vitro studies provide important results helping in designing clinical trials along with other advantageous aspects like a lack of ethical concerns and economic constraints. ${ }^{11,39}$ Besides, it is important to conduct in vitro studies that assess other relatable outcomes, such as canal position, instrumentation efficacy and disinfection of the root canal before running clinical trials in order to prevent treatment failure and subsequently clinical harm to the research subjects.

\section{Conclusion}

The current findings in this systematic review do not lead us to a conclusive and clear outcome due to the restricted number of studies. Thus, it requires more confirmation and clarification by performing other studies with larger amounts of examined cases and having them be randomized clinical trials in particular. In addition, it is important to mention that studying disinfection efficiency along with the conservative endodontic cavity is essential before starting the clinical trial in order to prevent harm to the patient. For more support and emphasis, retrospective and prospective studies must be initialized. In sum of this systematic review, no final decision could be made whether ConsAC is more advantageous than TradAC in fracture resistance when examined in human molars and further studies are needed.

\section{Acknowledgment}

We would like to thank Ajman University for facilitating our work and their continuous help and support.

\section{Disclosure}

All authors declare no financial or other conflicts of interest.

\section{References}

1. Varghese VS, George JV, Mathew S, Nagaraja S, Indiresha HN, Madhu KS. Cone beam computed tomographic evaluation of two access cavity designs and instrumentation on the thickness of peri-cervical dentin in mandibular anterior teeth. $J$ Conserv Dent. 2016;19(5):450. doi:10.4103/0972-0707.190018 
2. Jiang Q, Huang Y, Tu X, Li Z, He Y, Yang X. Biomechanical properties of first maxillary molars with different endodontic cavities: a finite element analysis. J Endod. 2018;44(8):1283-1288.

3. Mittal A, Dadu S, Garg P, Yendrembam B, Abraham A, Singh K. Comparative evaluation of fracture resistance of endodontically treated teeth with epoxy resin-based sealers AH plus and mineral trioxide aggregate fillapex: an in vitro study. Indian J Dent Sci. 2017;9(1):8. doi:10.4103/IJDS.IJDS_83_16

4. Neelakantan P, Khan K, Ng GP, Yip CY, Zhang C, Cheung GS. Does the orifice-directed dentin conservation access design debride pulp chamber and mesial root canal systems of mandibular molars similar to a traditional access design? J Endod. 2018;44(2):274-279. doi:10.1016/j.joen.2017.10.010

5. Osman IA, Ahmed HA. The effect of access cavity design on fracture resistance of endodontically treated first molars: in vitro study. Sch J Dent Sci. 2018;5(9):443-451. doi:10.21276/sjds.2018.5.9.8

6. Caplan DJ, Cai J, Yin G, White BA. Root canal filled versus non-root canal filled teeth: a retrospective comparison of survival times. $J$ Public Health Dent. 2005;65(2):90-96. doi:10.1111/j.17527325.2005.tb02792.x

7. Vire DE. Failure of endodontically treated teeth: classification and evaluation. $J$ Endod. 1991;17(7):338-342. doi:10.1016/S0099. 2399(06)81702-4

8. Kishen A. Mechanisms and risk factors for fracture predilection in endodontically treated teeth. Endod Topics. 2006;13(1):57-83. doi:10.1111/j.1601-1546.2006.00201.x

9. Clark D, Khademi JA. Case studies in modern molar endodontic access and directed dentin conservation. Dent Clin N Am. 2010;54 (2):275-289. doi:10.1016/j.cden.2010.01.001

10. Al Amri MD, Al-Johany S, Sherfudhin H, et al. Fracture resistance of endodontically treated mandibular first molars with conservative access cavity and different restorative techniques: an in vitro study. Aust Endod J. 2016;42(3):124-131. doi:10.1111/aej.12148

11. Silva EJ, Rover G, Belladonna FG, De-Deus G, da Silveira Teixeira C, da Silva Fidalgo TK. Impact of contracted endodontic cavities on fracture resistance of endodontically treated teeth: a systematic review of in vitro studies. Clin Oral Invest. 2018;22 (1):109-118. doi:10.1007/s00784-017-2268-y

12. Bóveda C, Kishen A. Contracted endodontic cavities: the foundation for less invasive alternatives in the management of apical periodontitis. Endod Topics. 2015;33(1):169-186. doi:10.1111/ etp. 12088

13. Chlup Z, Žižka R, Kania J, Přibyl M. Fracture behaviour of teeth with conventional and mini-invasive access cavity designs. J Eur Ceram Soc. 2017;37(14):4423-4429. doi:10.1016/j.jeurceramsoc.2017.03.025

14. Moore B, Verdelis K, Kishen A, Dao T, Friedman S. Impacts of contracted endodontic cavities on instrumentation efficacy and biomechanical responses in maxillary molars. $J$ Endod. 2016;42 (12):1779-1783. doi:10.1016/j.joen.2016.08.028

15. Pantvisai P, Messer HH. Cuspal deflection in molars in relation to endodontic and restorative procedures. J Endod. 1995;21(2):57-61. doi:10.1016/S0099-2399(06)81095-2

16. Soares PV, Santos-Filho PC, Gomide HA, Araujo CA, Martins LR, Soares CJ. Influence of restorative technique on the biomechanical behavior of endodontically treated maxillary premolars: part II: strain measurement and stress distribution. J Prosthet Dent. 2008;99 (2):114-122. doi:10.1016/S0022-3913(08)60027-X

17. Pereira JR, McDonald A, Petrie A, Knowles JC. Effect of cavity design on tooth surface strain. J Prosthet Dent. 2013;110(5):369-375. doi:10.1016/j.prosdent.2013.08.004

18. Lang H, Korkmaz Y, Schneider K, Raab WM. Impact of endodontic treatments on the rigidity of the root. J Dent Res. 2006;85 (4):364-368.

19. Clark D, Khademi J, Herbranson E. Fracture resistant endodontic and restorative preparations. Dent Today. 2013;32(2):118-120.
20. Yuan K, Niu C, Xie Q. Comparative evaluation of the impact of minimally invasive preparation vs. conventional straight-line preparation on tooth biomechanics: a finite element analysis. Eur J Oral Sci. 2016;124(6):591-596. doi:10.1111/eos.12303

21. Sabeti M, Kazem M, Dianat O, et al. Impact of access cavity design and root canal taper on fracture resistance of endodontically treated teeth: an ex vivo investigation. J Endod. 2018;44(9):1402-1406. doi:10.1016/j.joen.2018.05.006

22. Silva EJNL, Pinto KP, Ferreira CM, et al. Current status on minimal access cavity preparations: a critical analysis and a proposal for a universal nomenclature. Int Endod J. 2020. doi:10.1111/iej.13391.

23. Sarkis-Onofre R, Skupien JA, Cenci MS, Moraes RR, PereiraCenci T. The role of resin cement on bond strength of glass-fiber posts luted into root canals: a systematic review and meta-analysis of in vitro studies. Oper Dent. 2014;39(1):E31-44. doi:10.2341/13070-LIT

24. da Rosa WL, Piva E, da Silva AF. Bond strength of universal adhesives: a systematic review and meta-analysis. J Dent. 2015;43 (7):765-776. doi:10.1016/j.jdent.2015.04.003

25. Moher D, Liberati A, Tetzlaff J, Altman DG, The PRISMA Group (2009). Preferred Reporting Items for Systematic Reviews and MetaAnalyses: The PRISMA Statement. PLoS Med 6(7): e1000097. doi:10.1371/journal.pmed1000097

26. Corsentino G, Pedullà E, Castelli L, et al. Influence of access cavity preparation and remaining tooth substance on fracture strength of endodontically treated teeth. $J$ Endod. 2018;44(9):1416-1421. doi:10.1016/j.joen.2018.05.012

27. Makati D, Shah NC, Brave D, Rathore VP, Bhadra D, Dedania MS. Evaluation of remaining dentin thickness and fracture resistance of conventional and conservative access and biomechanical preparation in molars using cone-beam computed tomography: an in vitro study. J Conserv Dent. 2018;21(3):324. doi:10.4103/JCD.JCD_311_17

28. Plotino G, Grande NM, Isufi A, et al. Fracture strength of endodontically treated teeth with different access cavity designs. $J$ Endod. 2017;43(6):995-1000. doi:10.1016/j.joen.2017.01.022

29. Krishan R, Paqué F, Ossareh A, Kishen A, Dao T, Friedman S. Impacts of conservative endodontic cavity on root canal instrumentation efficacy and resistance to fracture assessed in incisors, premolars, and molars. J Endod. 2014;40(8):1160-1166. doi:10.1016/j. joen.2013.12.012

30. Rover G, Belladonna FG, Bortoluzzi EA, De-Deus G, Silva EJ, Teixeira CS. Influence of access cavity design on root canal detection, instrumentation efficacy, and fracture resistance assessed in maxillary molars. J Endod. 2017;43(10):1657-1662. doi:10.1016/j. joen.2017.05.006

31. Maske A, Weschenfelder VM. Influence of access cavity design on fracture strength of endodontically treated lower molars. Aust Endod J. 2020. doi:10.1111/aej.12446

32. Barbosa AFA, Silva EJNL, Coelho BP, Ferreira CMA, Lima CO, Sassone LM. The influence of endodontic access cavity design on the efficacy of canal instrumentation, microbial reduction, root canal filling and fracture resistance in mandibular molars. Int Endod J. 2020. doi:10.1111/iej.13383

33. Hamouda IM, Shehata SH. Fracture resistance of posterior teeth restored with modern restorative materials. J Biomed Res. 2011;25 (6):418-424. doi:10.1016/S1674-8301(11)60055-9

34. Özyürek T, Ö Ü, EÖ D, Y1lmaz F. The effects of endodontic access cavity preparation design on the fracture strength of endodontically treated teeth: traditional versus conservative preparation. $J$ Endod. 2018;44(5):800-805. doi:10.1016/j.joen.2018.01.020

35. Buchanan L. Cutting endodontic access cavities-for long-term outcomes. Dental- tribune; 2017. Available from: http://www.dentaltri bune.com/articles/specialities/endodontics/33170_cutting_endodon tic_access_cavities_for_longterm_outcomes.html. 
36. Evans D. Hierarchy of evidence: a framework for ranking evidence evaluating healthcare interventions. J Clin Nurs. 2003;12(1):77-84. doi:10.1046/j.1365-2702.2003.00662.x

37. Murdoch-Kinch CA, McLEAN ME. Minimally invasive dentistry. $J$ Am Dent Assoc. 2003;134(1):87-95. doi:10.14219/jada. archive.2003.0021
38. Collier R. Rapidly rising clinical trial costs worry researchers. Can Med Assoc J. 2009;180(3):277-278. doi:10.1503/cmaj.082041

39. Fröhlich E, Salar-Behzadi S. Toxicological assessment of inhaled nanoparticles: role of in vivo, ex vivo, in vitro, and in silico studies. Int $\mathrm{J} \mathrm{Mol}$ Sci. 2014;15(3):4795-4822. doi:10.3390/ijms 15034795

\section{Publish your work in this journal}

Clinical, Cosmetic and Investigational Dentistry is an international, peer-reviewed, open access, online journal focusing on the latest clinical and experimental research in dentistry with specific emphasis on cosmetic interventions. Innovative developments in dental materials, techniques and devices that improve outcomes and patient satisfaction and preference will be highlighted. The manuscript management system is completely online and includes a very quick and fair peer-review system, which is all easy to use. Visit http://www.dovepress.com/testimonials.php to read real quotes from published authors. 